\title{
Cascaded third harmonic generation in lithium niobate nanowaveguides
}

\author{
Alexander S. Solntsev, ${ }^{1, a)}$ Andrey A. Sukhorukov, ${ }^{1}$ Dragomir N. Neshev, ${ }^{1}$ Rumen lliew, ${ }^{2}$ \\ Reinhard Geiss, ${ }^{3}$ Thomas Pertsch, ${ }^{3}$ and Yuri S. Kivshar ${ }^{1}$ \\ ${ }^{1}$ Nonlinear Physics Centre and Centre for Ultra-high bandwidth Devices for Optical Systems (CUDOS), \\ Research School of Physics and Engineering, Australian National University, Canberra ACT \\ 0200, Australia \\ ${ }^{2}$ Institute of Condensed Matter Theory and Solid State Optics, Friedrich-Schiller-Universität Jena, \\ Max-Wien-Platz 1, 07743 Jena, Germany \\ ${ }^{3}$ Institute of Applied Physics, Friedrich-Schiller-Universität Jena, Max-Wien-Platz, 1, 07743 Jena, Germany
}

(Received 27 March 2011; accepted 12 May 2011; published online 9 June 2011)

\begin{abstract}
We predict highly efficient third harmonic generation through simultaneous phase-matching of second-harmonic generation and sum-frequency generation in lithium niobate nanowaveguides, enabled due to strong modal dispersion. We demonstrate that the waveguide size which corresponds to phase-matching is also optimal for highest mode confinement and therefore for strongly enhanced conversion efficiency. (C) 2011 American Institute of Physics. [doi:10.1063/1.3597627]
\end{abstract}

Parametric frequency conversion and harmonic generation are among the most intensively studied phenomena in nonlinear optics. It was demonstrated that important advantages can be obtained by cascading several parametric processes. ${ }^{1}$ In particular, the highest efficiency third harmonic generation (THG) can be achieved in media with quadratic nonlinearity through two cascaded parametric processes: (i) second harmonic generation (SHG) and (ii) sumfrequency generation (SFG) that incorporates mixing of the fundamental wave $(\mathrm{FW})$ and the second harmonic $(\mathrm{SH})$ to generate the third harmonic (TH). The conversion is most efficient when the phase-matching (PM) conditions for both processes are satisfied.

Whereas material birefringence can be conveniently used to phase-match a single process, ${ }^{2}$ the simultaneous PM of cascaded processes requires engineering of linear or nonlinear material properties at microscale and nanoscale. In particular, quasi-PM (Ref. 3) of cascaded THG (Refs. 4 and 5) can be realized by dual-period inversion of the crystal domain structure through electric field poling of the crystal.

With the development of nanotechnology, it became possible to fabricate high-index-contrast nanowaveguides which provide strong spatial confinement of the guided modes thus enhancing the nonlinear interactions. By using such waveguides one can achieve PM of quadratic nonlinear processes through dispersion engineering of guided modes. ${ }^{6-10}$ This is a flexible approach that avoids the need for additional periodic poling of the waveguide and has been widely used for PM of four-wave mixing nonlinear interactions. ${ }^{11}$

In this letter, we predict that ultrathin $\mathrm{LiNbO}_{3}$ waveguides suspended in air can be used for efficient cascaded THG. Air suspended $\mathrm{LiNbO}_{3}$ slab waveguides were shown to be feasible for fabrication at various thicknesses ${ }^{12,13}$ and with higher quality in comparison to epitaxially grown $\mathrm{LiNbO}_{3}$ films. ${ }^{14}$ The $\mathrm{SHG}$ in $\mathrm{LiNbO}_{3}$ material was studied theoretically in photonic crystals ${ }^{10}$ and thin films on a substrate. ${ }^{7}$ Furthermore efficient SHG was also predicted for AlGaAs slot waveguides, ${ }^{6}$ rods, ${ }^{8}$ and thin highindex-contrast AlGaAs/oxidized AlAs waveguides. ${ }^{9}$ How-

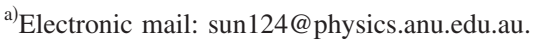

ever, the simultaneous PM of cascaded parametric processes in nanowaveguides has never been considered. We predict that complete PM of both cascaded quadratic processes can be realized simultaneously in a single waveguide without periodic poling. The high quadratic nonlinearity of $\mathrm{LiNbO}_{3}$ and tight field confinement in a nanowaveguide can allow for a strong enhancement of the conversion efficiency in comparison to quasiphase-matched cascaded THG in periodically poled $\mathrm{LiNbO}_{3}$ waveguides ${ }^{1,5}$ and direct THG through cubic nonlinearity in silica glass waveguides. ${ }^{15}$

To get a better insight in the possibilities for PM of the cascaded THG, we first develop a semianalytical model that describes $\mathrm{PM}$ in $\mathrm{LiNbO}_{3}$ slab waveguides. We then show that this model can also be used as a starting point to design more practical nanowire waveguides. For efficient frequency conversion, in addition to PM, it is desirable to employ nonlinear interactions based on the largest nonlinear susceptibility tensor components. We, therefore, perform a systematic analysis of different slab orientations, and find that the most efficient cascaded THG is realized in a $z$-cut $\mathrm{LiNbO}_{3}$ slab waveguide with all interacting waves propagating in $y$ direction, as illustrated in Fig. 1(a).

We determine that the optimal THG process in the $\mathrm{LiNbO}_{3}$ slab waveguide is the conversion of $\mathrm{FW} \mathrm{TE}$ mode into $\mathrm{SH} \mathrm{TM}_{0}$ mode, and then their mixing to the $\mathrm{TH} \mathrm{TE}_{1}$ mode in the process of SFG [see Fig. 1(b)]. The electric field profiles of the guided modes can be expressed as $\mathbf{E}$ $=\exp \left[-i \omega t+i k_{0} n_{\mathrm{eff}}(\omega) y\right] \times\left[E_{x}(z, \omega), E_{y}(z, \omega), E_{z}(z, \omega)\right]$. Here $n_{\text {eff }}$ is the effective mode index, $\omega$ is the fundamental optical frequency, $k_{0}=\omega / c$, and $c$ is the speed of light in vacuum. For TE modes, the electric field is polarized in the $x$ direction $\left(E_{z}=E_{y}=0\right)$, and the electric field component $E_{x}$ satisfies the wave equation: $\partial^{2} E_{x} / \partial z^{2}=E_{x} k_{0}^{2}\left[\left(n_{\text {eff }}^{\mathrm{TE}}\right)^{2}-n_{x}^{2}\right]$, where $n_{x, y, z}$ are refractive index components, equal to those of congruent $\mathrm{LiNbO}_{3}$ (Ref. 16) for $|z| \leq h / 2$ (inside the slab waveguide), and $n_{x, y, z}=1$ for $|z|>h / 2$ (outside the slab waveguide), where $h$ is the waveguide thickness. For TM modes, the electric field is polarized in the $y-z$ plane and can be expressed through the single component of the magnetic field $H_{x}, E_{z}$ $=-Z_{0} n_{\mathrm{eff}}^{\mathrm{TM}} n_{z}^{-2} H_{x}$ and $E_{y}=-i Z_{0} k_{0}^{-1} n_{y}^{-2} \partial H_{x} / \partial z$ with the vacuum impedance $Z_{0}=\left(\mu_{0} / \varepsilon_{0}\right)^{1 / 2}$. The wave equation for the magnetic field is $n_{z}^{2} \partial\left[n_{y}^{-2} \partial H_{x} / \partial z\right] / \partial z=H_{z} k_{0}^{2}\left[\left(n_{\text {eff }}^{\mathrm{TM}}\right)^{2}-n_{z}^{2}\right]$. 


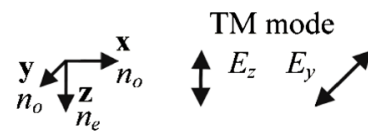

TE mode $E_{x} \leftrightarrow$

(a)

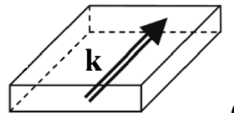

(c)

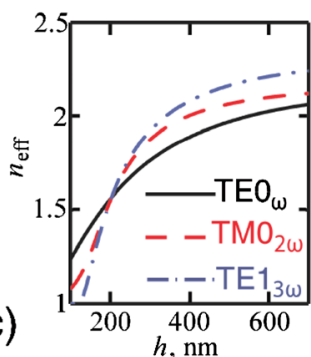

(b)
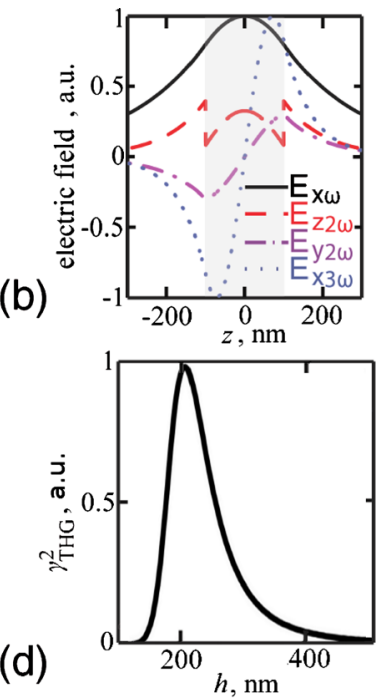

FIG. 1. (Color online) (a) $\mathrm{LiNbO}_{3}$ slab waveguide orientation for THG. (b) Electric field profiles for slab waveguide thickness of $200 \mathrm{~nm}$ and FW wavelength of $1542 \mathrm{~nm}$. (c) Effective refractive index vs slab waveguide thickness for FW, SH, and TH modes. (d) Normalized square of THG nonlinear coefficient for slab waveguide.

We solve the wave equations using the transfer matrix method $^{17}$ and obtain the effective indices and profiles of the guided modes. Then, the frequency conversion can be modeled by coupled equations for the amplitudes of narrowband FW, SH, and TH modes, ${ }^{1} U, V$, and $W$, respectively. In the case of undepleted pump approximation, $U \gg V \gg W$, the coupled equations reduce to $\partial V / \partial y=\gamma_{\mathrm{SHG}} U^{2} \exp \left(i \Delta k_{\mathrm{SHG}} y\right)$ and $\quad \partial W / \partial y=\gamma_{\mathrm{SFG}} V U \exp \left(i \Delta k_{\mathrm{SFG}} y\right)$. Here $\Delta k_{\mathrm{SHG}}=2\left(n_{\mathrm{FW}}\right.$ $\left.-n_{\mathrm{SH}}\right) k_{0}, \Delta k_{\mathrm{SFG}}=\left(3 n_{\mathrm{TH}}-2 n_{\mathrm{SH}}-n_{\mathrm{FW}}\right) k_{0}$ are the wave number mismatches, $\quad n_{\mathrm{FW}}=n_{\mathrm{eff}}^{\mathrm{TE}}(\omega), \quad n_{\mathrm{SH}}=n_{\mathrm{eff}}^{\mathrm{TM}}(2 \omega), \quad n_{\mathrm{TH}}=n_{\mathrm{eff}}^{\mathrm{TE}_{1}}(3 \omega)$, where $n_{\mathrm{eff}}^{\mathrm{TE}}$ is the effective index of $\mathrm{TE}_{1}$ mode. Employing the approach developed for SHG in nanostructured waveguides, ${ }^{10}$ we derive an expression for the nonlinear coefficients for SHG and SFG

$$
\begin{aligned}
& \gamma_{\mathrm{SHG}}=\omega d_{31} \sqrt{\frac{2 n_{\mathrm{g}, \mathrm{FW}}^{2} n_{\mathrm{g}, \mathrm{SH}}}{n_{\mathrm{FW}}^{4} n_{\mathrm{SH}}^{2} \varepsilon_{0} c^{3} A_{\mathrm{SHG}}}}, \\
& \gamma_{\mathrm{SFG}}=\omega d_{16} \sqrt{\frac{18 n_{\mathrm{g}, \mathrm{FW}} n_{\mathrm{g}, \mathrm{SH}} n_{\mathrm{g}, \mathrm{TH}}}{n_{\mathrm{FW}}^{2} n_{\mathrm{SH}}^{2} n_{\mathrm{TH}}^{2} \varepsilon_{0} c^{3} A_{\mathrm{SFG}}}},
\end{aligned}
$$

where $\quad n_{\mathrm{g}, \mathrm{FW}}=\partial\left[\omega n_{\mathrm{eff}}^{\mathrm{TE}}(\omega)\right] / \partial \omega, \quad n_{\mathrm{g}, \mathrm{SH}}=\partial\left[\omega n_{\mathrm{eff}}^{\mathrm{TM}}(2 \omega)\right] / \partial \omega$, $n_{\mathrm{g}, \mathrm{TH}}=\partial\left[\omega n_{\mathrm{eff}}^{\mathrm{TE}_{1}}(3 \omega)\right] / \partial \omega$ are the group indices which define the group velocity $V_{\mathrm{g}}=c / n_{\mathrm{g}}, d_{31}$ and $d_{16}$ are the nonlinear optical coefficients of $\mathrm{LiNbO}_{3}$. The normalized effective areas for SHG and SFG are

$$
\begin{aligned}
A_{\mathrm{SHG}} & =\frac{\left(\int \mathrm{d} x \mathrm{~d} z E_{\mathrm{FW}}^{2}\right)^{2} \int \mathrm{d} x \mathrm{~d} z E_{\mathrm{SH}}^{2}}{\left|\int_{\mathrm{NL}} \mathrm{d} x \mathrm{~d} z E_{z}^{*}(x, z, 2 \omega) E_{x}^{2}(x, z, \omega)\right|^{2}}, \\
A_{\mathrm{SFG}} & =\frac{\int \mathrm{d} x \mathrm{~d} z E_{\mathrm{FW}}^{2} \int \mathrm{d} x \mathrm{~d} z E_{\mathrm{SH}}^{2} \int \mathrm{d} x \mathrm{~d} z E_{\mathrm{TH}}^{2}}{\left|\int_{\mathrm{NL}} \mathrm{d} x \mathrm{~d} z E_{\mathrm{TH}} E_{x}^{*}(x, z, \omega) E_{y}^{*}(x, z, 2 \omega)\right|^{2}},
\end{aligned}
$$

where $E_{\mathrm{FW}}=E_{x}(x, z, \omega), E_{\mathrm{SH}}=\left[E_{z}^{2}(x, z, 2 \omega)+E_{y}^{2}(x, z, 2 \omega)\right]^{1 / 2}$, $E_{\mathrm{TH}}=E_{x}^{\mathrm{TE}_{1}}(x, z, 3 \omega)$ are $\mathrm{FW}, \mathrm{SH}$, and TH electric field absolute values, NL is a waveguide cross-section area, and * stands for complex conjugation. For slab waveguides, the result of the integration over $x$ will depend on the input beam width and profile.

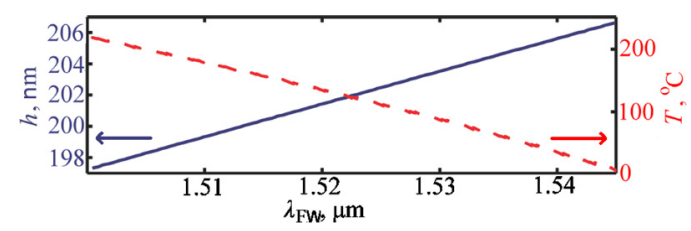

FIG. 2. (Color online) The slab waveguide thickness (solid line, left axis) and temperature (dashed line, right axis) corresponding to exact PM for THG vs FW wavelength.

The highest THG efficiency is realized when SHG and SFG processes are phase-matched simultaneously. In Fig. 1(c) we show that the effective refractive indices for all three harmonics with $\mathrm{FW}$ wavelength $\lambda_{\mathrm{FW}}=1542 \mathrm{~nm}$ are matched for a slab waveguide thickness of $h=206 \mathrm{~nm}$. In this case the normalized conversion efficiencies for SHG and SFG in the undepleted narrow band pump approximation can be defined as $\eta_{\mathrm{SHG}}=\gamma_{\mathrm{SHG}}^{2}$ and $\eta_{\mathrm{SFG}}=\gamma_{\mathrm{SFG}}^{2}$. We estimate these efficiencies for the beam width equal to $1.77 \mu \mathrm{m}$ in $x$ direction (for the later comparison with fully vectorial calculation for nanowire waveguides): $\eta_{\mathrm{SHG}}=347 \% \mathrm{~W}^{-1} \mathrm{~cm}^{-2}$, $\eta_{\mathrm{SFG}}=215 \% \mathrm{~W}^{-1} \mathrm{~cm}^{-2}$. Total normalized conversion efficiency for phase-matched THG in a waveguide length $L$ can be defined as $\eta_{\mathrm{THG}}=\gamma_{\mathrm{THG}}^{2}=|W(L)|^{2} /\left(|U|^{4} L^{2}\right)$ $=\eta_{\mathrm{SHG}} \eta_{\mathrm{SFG}}|U|^{2} L^{2} / 4$. It is most remarkable that the slab waveguide thickness of $h=206 \mathrm{~nm}$ also corresponds to the strongest mode confinement and therefore to the highest nonlinear coefficient for THG, as shown in Fig. 1(d).

We find that both PM conditions can be satisfied for different FW wavelengths by manufacturing a slab waveguide with appropriate thickness and by heating or cooling the sample, see Fig. 2. Furthermore, it is possible to engineer the ratio $\gamma_{\mathrm{SHG}} / \gamma_{\mathrm{SFG}}$, which can enable nearly complete conversion of energy into $\mathrm{TH} .^{18}$

We now consider a nanowire waveguide with rectangular cross-section [see Fig. 3(a)], in which higher conversion efficiency can be achieved due to mode confinement in both transverse dimensions $(z, x)$. We calculate numerically the FW, SH, and TH modes in nanowaveguides with a rectangular cross-section using the COMSOL rf module. We start with crystal orientation and waveguide thickness $h$ as in the slab waveguide analysis and then optimize the FW frequency and
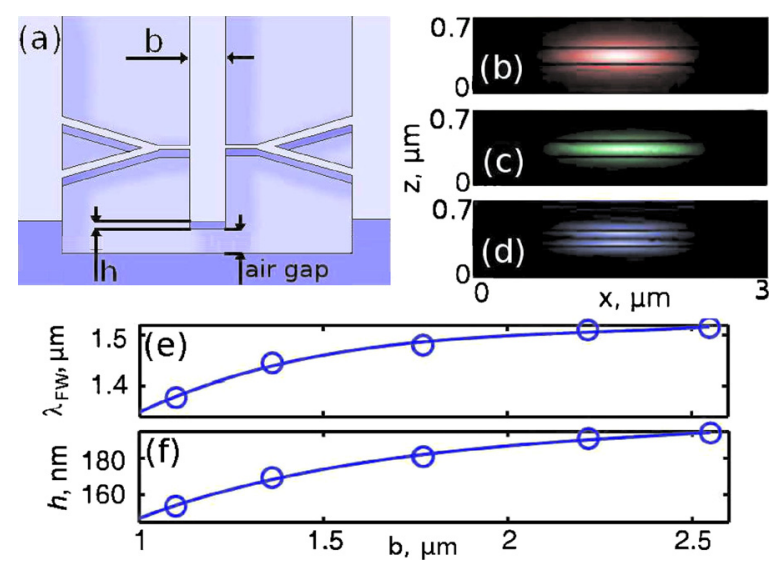

FIG. 3. (Color online) (a) Scheme of the air-suspended $\mathrm{LiNbO}_{3}$ waveguide. [(b)-(d)] Intensity profiles of phase-matched (b) FW, (c) SH, (d) TH waves for a waveguide width $b=1.77 \mu \mathrm{m}$ and thickness $h=181 \mathrm{~nm}$. (e) The FW wavelength $\lambda_{\mathrm{FW}}$ and (f) the waveguide thickness $h$ corresponding to exact PM for THG vs waveguide width $b$. 
waveguide thickness to achieve complete PM. Characteristic modes of the interacting $\mathrm{FW}, \mathrm{SH}$, and $\mathrm{TH}$ waves in the waveguide are shown in Figs. 3(b)-3(d). We demonstrate in Figs. 3(e) and 3(f) that by varying the waveguide width $b$, we can satisfy the conditions for fully phase-matched THG for pump wavelengths between 1.35 and $1.55 \mu \mathrm{m}$. Using the calculated mode profiles we obtain the effective areas and the conversion efficiency for the cascaded THG. For $\lambda_{\mathrm{FW}}=1471 \mathrm{~nm}$, a waveguide of width $b$ $=1.77 \mu \mathrm{m}$ and thickness $h=181 \mathrm{~nm}$, the effective areas are $A_{\mathrm{SHG}}=22 \mu \mathrm{m}^{2}$ and $A_{\mathrm{SFG}}=26 \mu \mathrm{m}^{2}$. This corresponds to normalized conversion efficiencies $\eta_{\mathrm{SHG}}=261 \% \mathrm{~W}^{-1} \mathrm{~cm}^{-2}$, $\eta_{\mathrm{SFG}}=279 \% \mathrm{~W}^{-1} \mathrm{~cm}^{-2}$. We note that these values agree well with our previous estimates based on semianalytical analysis for a slab waveguide. Total THG conversion efficiency $\eta_{\mathrm{THG}}$ is sufficiently high to predict, in an ideal waveguide with a $3 \mathrm{~mm}$ interaction length, the generation of $1 \mathrm{~mW}$ green light from $33 \mathrm{~mW}$ source at a telecommunication wavelength. To compare with the best result reported to date, such $\mathrm{TH}$ output from ideal periodically poled $\mathrm{LiNbO}_{3}$ structure is predicted to require ten times longer crystal and almost four times higher pump power. ${ }^{5}$

The realization of $\mathrm{LiNbO}_{3}$ nanowaveguides with the desired geometry is feasible by means of ion-beam enhanced etching (IBEE). The IBEE technology has been shown to be ideally suited for the fabrication of complex photonic crystal structures $^{19,20}$ and can also be applied to fabricate the proposed nanowaveguides. In this process the geometry of a patterned masking layer is transferred into the crystal by a series of high energy ion irradiation. The irradiated and thus damaged regions are subsequently removed by chemical wet etching, resulting in an air-bridged structure, as shown schematically in Fig. 3(a). We note that complete PM requires high manufacturing precision. The thickness of $100 \mu \mathrm{m}$ long slab waveguides has to be controlled with nanometer accuracy, which may be challenging with technologies currently available. Nevertheless, THG may be realized even when the individual SHG and SFG processes are slightly mismatched, provided that a single PM condition between the FW and TH waves is satisfied. ${ }^{1}$ We anticipate that broadband THG may be possible in the latter case, although at the trade-off of lower conversion efficiency.
In conclusion, we have shown that the modal dispersion in air-suspended lithium niobate waveguides can be used to simultaneously phase-match two different nonlinear parametric processes, resulting in cascaded THG. We anticipate that our findings may stimulate the realization of distinct families of efficient frequency mixers and converters to the $\mathrm{TH}$, for applications in compact light sources and laser display technologies.

We acknowledge the support by the Australian Research Council, the Australian Academy of Science, Australian National Computational Infrastructure, and German Research Foundation, Grant No. NanoGuide, PE 1524/5-1.

${ }^{1}$ S. M. Saltiel, A. A. Sukhorukov, and Yu. S. Kivshar, Prog. Opt. 47, 1 (2005).

${ }^{2}$ R. W. Boyd, Nonlinear Optics (Academic Press, San Diego, 1992).

${ }^{3}$ M. M. Fejer, G. A. Magel, D. H. Jundt, and R. L. Byer, IEEE J. Quantum Electron. 28, 2631 (1992).

${ }^{4}$ Y. Sheng, S. M. Saltiel, and K. Koynov, Opt. Lett. 34, 656 (2009).

${ }^{5}$ M. Marangoni, M. Lobino, and R. Ramponi, Opt. Lett. 31, 2707 (2006).

${ }^{6}$ A. Di Falco, C. Conti, and G. Assanto, Opt. Lett. 31, 3146 (2006).

${ }^{7}$ J. C. G. De Sande, S. Stivala, J. Gonzalo, and G. Assanto, J. Nonlinear Opt. Phys. Mater. 15, 191 (2006).

${ }^{8}$ A. M. Zheltikov, Opt. Commun. 270, 402 (2007).

${ }^{9}$ H. Ishikawa and T. Kondo, Appl. Phys. Express 2, 042202 (2009).

${ }^{10}$ R. Iliew, C. Etrich, T. Pertsch, F. Lederer, and Yu. S. Kivshar, Phys. Rev. A 81, 023820 (2010).

${ }^{11}$ M. A. Foster, A. C. Turner, J. E. Sharping, B. S. Schmidt, M. Lipson, and A. L. Gaeta, Nature (London) 441, 960 (2006).

${ }^{12}$ A. M. Radojevic, M. Levy, H. Kwak, and R. M. Osgood, Appl. Phys. Lett. 75, 2888 (1999).

${ }^{13}$ F. Schrempel, T. Gischkat, H. Hartung, T. Hoche, E. B. Kley, A. Tunnermann, and W. Wesch, Opt. Lett. 34, 1426 (2009).

${ }^{14}$ X. Lansiaux, E. Dogheche, D. Remiens, M. Guilloux viry, A. Perrin, and P. Ruterana, J. Appl. Phys. 90, 5274 (2001).

${ }^{15}$ N. Y. Joly, T. A. Birks, A. Yulin, J. C. Knight, and P. S. J. Russell, Opt. Lett. 30, 2469 (2005).

${ }^{16}$ G. J. Edwards and M. Lawrence, Opt. Quantum Electron. 16, 373 (1984).

${ }^{17}$ M. Born and E. Wolf, Principles of Optics: Electromagnetic Theory of Propagation, Interference and Diffraction of Light, 7th ed. (Cambridge University Press, UK, 2002).

${ }^{18}$ O. A. Egorov and A. P. Sukhorukov, Bull. Russ. Acad. Sci. Phys. 62, 1884 (1998)

${ }^{19}$ H. Hartung, E. B. Kley, T. Gischkat, F. Schrempel, W. Wesch, and A. Tunnermann, Opt. Mater. (Amsterdam, Neth.) 33, 19 (2010).

${ }^{20}$ R. Geiss, S. Diziain, R. Iliew, C. Etrich, H. Hartung, N. Janunts, F. Schrempel, F. Lederer, T. Pertsch, and E. B. Kley, Appl. Phys. Lett. 97 131109 (2010). 